Article

\title{
Functional Polyimide-Based Electrospun Fibers for Biomedical Application
}

\author{
Diana Serbezeanu ${ }^{1, *}$, Tăchiță Vlad-Bubulac ${ }^{1} \mathbb{D}$, Daniela Rusu ${ }^{1}$, \\ Grațiela Grădișteanu Pircalabioru ${ }^{2}$, Iuliana Samoilă ${ }^{3}$ (D), Sorina Dinescu ${ }^{3}$ (D) \\ and Magdalena Aflori ${ }^{1, * \mathbb{D}}$ \\ 1 "Petru Poni" Institute of Macromolecular Chemistry, Aleea Grigore Ghica Voda, 41A, 700487 Iasi, Romania; \\ tvladb@icmpp.ro (T.V.-B.); rusu.daniela@icmpp.ro (D.R.) \\ 2 Sanimed International IMPEX SRL, Sos. București Măgurele 70F, 051434 Bucharest, Romania; \\ gratiela87@gmail.com \\ 3 Department of Biochemistry and Molecular Biology, University of Bucharest, 91-95 Splaiul Independentei, \\ 050095 Bucharest, Romania; iuliana.samoila@bio.unibuc.ro (I.S.); sorina.dinescu@bio.unibuc.ro (S.D.) \\ * Correspondence: diana.serbezeanu@icmpp.ro (D.S.); maflori@icmpp.ro (M.A.)
}

Received: 31 August 2019; Accepted: 27 September 2019; Published: 29 September 2019

check for updates

\begin{abstract}
The current study focuses on the application of cytotoxicity tests upon one membrane matrix based on electrospun polyimide fibers, appealing for biomedical application, such as scaffolds for cell growth, patches or meshes for wound healing, etc. Assays were performed in order to determine the viability and proliferation of L929 murine fibroblasts after they were kept in direct contact with the studied electrospun polyimide fibers. Increased cell viability and proliferation were detected for cells seeded on electrospun polyimide fibers membrane, in comparison with the control system, either after two or six days of evaluation. The number of live cells was higher on the studied material compared to the control, after two and six days of cell seeding. The tendency of the cells to proliferate on the electrospun polyimide fibers was revealed by confocal microscopy. The morphological stability of electrospun polyimide membrane was evaluated by SEM observation, after immersion of the samples in phosphate buffer saline solution (PBS, 7.4 at $37^{\circ} \mathrm{C}$ ) at various time intervals. Additionally, the easy production of electrospun polyimide fibers can facilitate the development of these types of matrices into specific biomedical applications in the future.
\end{abstract}

Keywords: electrospun polyimide fibers; dermal fibroblasts; MTT test; Live-Dead test; antimicrobial properties; anti-biofilm activity

\section{Introduction}

Over the past few years, new worldwide public health challenges, such as antimicrobial resistance and biofilm formation were introduced into the agenda of some scientific communities, thus, governing the development of novel bioactive molecules capable of fighting against multidrug resistant bacteria [1-3]. Moreover, some studies revealed the approval of bio-medicinal herbal extracts or infusions of plants to deal with infectious pathogens in various diseases, opening the door for new development steps in medicine branches, such as skin regeneration, wound healing, tissue dressing, etc. For these types of applications, few groups used bioactive electrospun fibers as support matrix for their herbal extracts, in order to accelerate the healing/repair process of the treated tissue [4-8].

Electrospinning is an inexpensive and simple process to produce fibers using electrostatic force between surface charges to continuously draw nanofibers using a viscoelastic liquid. In the development of electrospun polymer fibers, diameter, and morphology can be controlled by a number of experimental parameters such as: Solution viscosity, charge density, surface tension, polymer molecular weight, 
dielectric constant, flow rate applied voltage, and tip-to-collector. Thus, nanofibers obtained using this technique exhibit a very large surface area, nanoscale structure and porosity, properties which recommend these electrospun fibers for a wide spectrum of application such as: environmental protection, water purification, air filtration, smart textiles, encapsulation of bioactive species, drug delivery, tissue engineering, and regenerative medicine [9-15].

An important class of high-performance polymers having excellent thermal, electrical, and mechanical properties, as well as remarkable chemical resistance is represented in the literature by aromatic polyimides [16-19]. Due to their outstanding performances, polyimides have been found very useful for numerous applications, for example, in ultra-large scale integrate circuit, multichip module packaging, printed circuit board fabrication [20-22], and lately they came into attention of the specialists from the biomedical field. For example, polyimide-based materials have been used for the construction of electrodes in biomedical microdevices [23,24] or for the manufacture of fibrous membranes operating as oxygenators in intravenous or intrathoracic devices, helping the optimal function of the lungs [25]. The biosafety of polyimides have been evidenced when this type of material was used for encapsulation of biosensors and more recently as a substrate for neuronal or ocular implants. Thus, in vitro tests indicated that polyimide implants were biocompatible, causing only moderate coagulation, minor cytotoxicity, and hemolysis [26], while supporting cell adhesion and cell growth in the presence of fibroblasts [27]. In vivo, polyimide implants showed good tissue integration and induced a very weak foreign body reaction, limited to a small area around the implants after an extended period of time [28]. Van Vlierberghe et al. have shown that following chemical modification the polyimides maintain their biocompatibility [29].

To date, electrospun nanofibers matrices appealing for the pharmaceutical industry have been studied, but to the best of our knowledge, reports about biological properties of electrospun fibers based on polyimide are rather rare in the literature. Electrospun polyimide fibers combine the advantages of polyimides, as a high-performance polymer class, with electrospinning process, as a tool for production of nanofiber membranes showing excellent properties, which attracted much attention from scientific worldwide communities [30-36]. Electrospun polyimide fibers have several characteristics such as large surface area to volume ratio, and a porous structure, due to the nanoscale network such as morphology. As the porosity of a scaffold is higher, the more homogeneous will be the cell distribution and interconnection for an engineered tissue [37,38]. These properties recommend polyimides nanofibers for a variety of biomedical applications such as drug delivery, medical implants, tissue engineering, and body-implant interphases [39]. Up to now, considerable efforts have been devoted to various electrospun polyimide fibers [30,40-42]. However, nearly no effort has been given to the development of electrospun polyimide fibers used in medical domain.

This work focuses on the synthesis of one polyimide bearing hydroxyl functional groups (DS-1). The polyimide DS- 1 has been synthesized by polycondensation reaction of appropriate monomers, followed by the production of fibers membranes based on the synthesized polyimide through the electrospinning procedure. The structural characterization and morphology of the electrospun polyimide fibers were investigated by FTIR, SEM and Raman, while its cytotoxicity was evaluated by the MTT assay and Live/Dead®(Molecular Probes, Eugene, OR, USA) assay. These tests were performed in contact with $\mathrm{L} 929$ murine fibroblasts at varying times. The high rates of viability and proliferation of cells in contact with the electrospun polyimide fibers shown by MTT and Live/Dead®tests demonstrated that this membrane is a biocompatible material. Antibacterial and anti-biofilm activities of electrospun polyimide fibers against Staphylococcus aureus and Pseudomonas aeruginosa strains were investigated.

\section{Experimental}

\subsection{Materials}

4-hydroxybenzaldehyde, aniline, aniline hydrochloride, 4,4'-oxydiphthalic anhydride (2), $\mathrm{N}$-methyl-2-pyrrolidone (NMP), $\mathrm{N}, \mathrm{N}$-dimethylformamide (DMF), and dimethyl sulfoxide (DMSO), 
were commercially obtained from Sigma-Aldrich (Saint Louis, MO, USA) and used as received. 4,4'-diamino-4"-hydroxy triphenylmethane was obtained by the reaction of 4-hydroxybenzaldehyde with aniline in the presence of an acid catalyst, as previously reported [43]. All other reagents and solvents were commercially available and were of analytical grade. 3-(4,5-Dimethyl-2-thiazolyl)-2,5diphenyl-2H-tetrazolium bromide (MTT), solution in PBS $(5 \mathrm{mg} / \mathrm{mL})$ were purchased from Sigma-Aldrich Chemie GmbH (Steinheim, Germany). LDH levels were quantified using the In Vitro Toxicology Assay Kit, Lactic Dehydrogenase based (Tox7 kit, Sigma-Aldrich). The Live/Dead kit was from ThermoFisher Scientific (Foster, CA, USA). The MTT solution was from Sigma-Aldrich Co, Steinheim, Germany. Bacterial strains (Staphylococcus aureus ATCC 25923, Pseudomonas aeruginosa ATCC 27853) and the L929 fibroblast cell line were purchased from ATCC (Manassas, VA, USA).

\subsection{Synthesis of DS-1}

The polyimide DS-1 was synthesized by a two-step polycondensation reaction using 4,4'-diamino-4"'-hydroxytriphenylmethane and 4,4'-oxydiphthalic anhydride [33]. Yield: $89 \%$. ATR-FTIR $\left(\mathrm{cm}^{-1}\right): v=3057$ (aromatic $\mathrm{C}-\mathrm{H}$ stretching vibration); $1773(\mathrm{C}=\mathrm{O}$ asymmetric stretching vibration); 1720 ( $\mathrm{C}=\mathrm{O}$ symmetric stretching vibration), 1607 and 1505 (aromatic $\mathrm{C}=\mathrm{C}$ ); 1368 (C-N stretching vibration); 745 (C-N ring deformation). ${ }^{1} \mathrm{H}$ NMR (DMSO- $\mathrm{d}_{6}, \delta(\mathrm{ppm})$ ): $9.35(1 \mathrm{H}, \mathrm{s})$; 8.07-8.05 (2H, d); $7.62(4 \mathrm{H}, \mathrm{s}) ; 7.41-7.29(8 \mathrm{H}, \mathrm{dd}) ; 7.03-7(2 \mathrm{H}, \mathrm{d}) ; 6.77-6.75(2 \mathrm{H}, \mathrm{d}) ; 5.66(1 \mathrm{H}, \mathrm{s})$.

\subsection{Fabrication of Electrospun Polyimide Fibers}

The polyimide $(23 \% \mathrm{w} / \mathrm{v})$ solution in DMF was fed from a $1 \mathrm{~mL}$ plastic syringe equipped with a 21-gauge stainless steel needle. The electric potential, obtained from a dual power source, was fixed at a positive output of $+10 \mathrm{kV}$ and a negative output of $-2 \mathrm{kV}$. The solution feed rate was set to $5 \mu \mathrm{L} / \mathrm{min}$. The electrospun polyimide fibers were collected on an anti-adhesive paper foil. The solution was electrospun at room temperature $\left(23 \pm 1^{\circ} \mathrm{C}\right)$. The collected electrospun polyimide fibers were allowed to dry under vacuum oven at $37^{\circ} \mathrm{C}$ [44].

\subsection{Characterization and Methods}

FTIR spectrum of electrospun polyimide fibers was obtained by using BioRad 'FTS 135' FTIR spectrometer equipped with a Specac "Golden Gate" ATR accessory. A LUMOS Microscope Fourier Transform Infrared (FTIR) spectrophotometer (Bruker Optik GmbH, Ettlingen, Germany), equipped with an attenuated total reflection (ATR) device was used to record the scans between 4000 and $500 \mathrm{~cm}^{-1}$ at a resolution of $4 \mathrm{~cm}^{-1}$.

${ }^{1} \mathrm{H}$ NMR spectrum was obtained on a Bruker Advance DRX 400 spectrometer, equipped with a $5 \mathrm{~mm}$, direct detection, multinuclear probe, operating at $400.1 \mathrm{MHz}$. The polymer sample was dissolved in DMSO and then measured at room temperature. ${ }^{1} \mathrm{H}$ chemical shifts $(\delta)$ were quoted in ppm relative to the residual peak of the solvent (ref. ${ }^{1} \mathrm{H}$ : DMSO- $d_{6}: 2.51 \mathrm{ppm}$ ).

The morphological characteristics of their surfaces were studied using a scanning electron microscope type Quanta 200 (FEI, Eindhoven, Netherland), operating at $20 \mathrm{kV}$ with secondary electrons in low vacuum mode, the magnification being indicated on each micrograph. The SEM studies were performed on uncoated samples fixed on aluminium substrate. The average fibers diameter was calculated from 42 fibers in three randomly selected areas using Scandium software on the SEM micrographs of the samples.

Raman spectra of electrospun polyimide fibers were recorded using an inVia ${ }^{\mathrm{TM}}$ confocal Raman microscope spectrometer (Renishaw plc, Gloucestershire, UK) equipped with a $633 \mathrm{~nm}$ excitation laser line and a Leica DM2700 microscope with $5 \times, 20 \times$ and 100× objectives and a Deep Depletion Renishaw CCD Centrus array detector. Scans were accumulated to obtain the spectra in the range of $100-4000 \mathrm{~cm}^{-1}$ with a resolution of $1 \mathrm{~cm}^{-1}$ and a laser power of $17 \mathrm{~mW}$. Electrospun polyimide fibers were measured directly from metal discs. 
Water uptake: To measure the water uptake, specimens of electrospun fibers (small square pieces $1 \mathrm{~cm} \times 1 \mathrm{~cm}$ ) were cut. These samples were immersed in $10 \mathrm{~mL}$ of distilled water, for different periods of time, from $10 \mathrm{~min}$ to $24 \mathrm{~h}$. Then, the samples were taken out and the excess surface water was quickly removed by blotting paper. The weight of the samples in wet state at each time point was noted using an electrical balance immediately. The degree of water uptake for every swelling period was calculated using the following formula:

$$
W U_{1-13}(\%)=\text { Wwet }_{1-13}-W d r y / W d r y \times 100
$$

where, Wwet ${ }_{1-13}$ is the wet weight of electrospun polyimide fibers at every swelling period $t_{1}$ to $t_{13}$, Wdry is the dry weight of electrospun polyimide fibers.

Static contact angle measurements were performed using a CAM 101 (KSV Instruments, Helsinki, Finland) system, equipped with a liquid dispenser, video camera, and drop shape analysis software. In order to obtain the statistical results, the experiments were conducted using double-distilled water on three different regions selected for the surface of the samples. All the measurements were performed at room temperature.

The morphological stability behavior was evaluated from the morphological change of the electrospun polyimide fibers. In this regard, the dried electrospun polyimide fibers were cut into small square pieces $(1 \mathrm{~cm} \times 1 \mathrm{~cm})$. Each cut of electrospun polyimide fibers membrane was exactly measured for initial weight and then immersed in $10 \mathrm{~mL}$ of phosphate buffer solution (PBS, pH 7.4 at $37^{\circ} \mathrm{C}$ ) for a period of two days and six days, respectively. When the predetermined time expired (two and six days), each sample of electrospun polyimide fibers membrane was extracted from vials, rinsed several times with distilled water to remove residual buffer salts, and dried to constant weight in an oven at $60^{\circ} \mathrm{C}$. The morphological change was estimated from the SEM observation as mentioned above, after immersion in PBS for two and six days, respectively.

Evaluation of the anti-biofilm effect of the electrospun-polyimide fibers membrane was performed following a protocol reported in the literature [45], as follows: Staphylococcus aureus ATCC 25923 and Pseudomonas aeruginosa ATCC 27853 strains were cultured on tryptic soy agar (Thermo Fisher Scientific, Waltham, MA, USA) for $18 \mathrm{~h}$ prior to the experiment. Subsequently, one colony was resuspended in $3 \mathrm{~mL}$ of tryptic soy broth media (MP Biomedicals, Solon, OH, USA) followed by incubation at $37^{\circ} \mathrm{C}$ (18 h, $200 \mathrm{rpm}$ ). The suspension was adjusted to an optical density of 0.52 (at OD $562 \mathrm{~nm}$ ) which corresponds to $10^{9}$ colony forming units (CFU) per mL. The inoculum was further diluted to $10^{6} \mathrm{CFU} / \mathrm{mL}$ in simulated body fluid supplemented with $10 \%$ fetal bovine serum. The samples for testing-tissue culture plastic control (TCPS), DS-1 and, silicone cathether were plated onto the wells of a 24-well plate. Next, $1 \mathrm{~mL}$ of the $10^{6} \mathrm{CFU} / \mathrm{mL}$ solution of P. aeruginosa/ S. aureus in simulated body fluid was pipetted into each well, while ensuring complete submersion of the samples. Samples were kept at $37^{\circ} \mathrm{C}$ at $5 \%$ carbon dioxide to allow biofilm formation. After $24 \mathrm{~h}$, excess bacteria were aspirated, and the samples were thoroughly washed with PBS (three times) to ensure the removal of all media residue and non-adherent bacteria. Samples were removed from the wells with a sterile forcep and immersed in $1 \mathrm{~mL}$ of PBS. To strip all adherent bacteria from the sample into solution, the tubes were vortexed at $3000 \mathrm{rpm}$ (20 $\mathrm{min})$. The resulting suspension was serially diluted in PBS in order to quantify the $\mathrm{CFU} / \mathrm{mL}$ values.

DS-1 biocompatibility tests were performed during one week of culture in standard conditions, by analyzing cell behavior in response to DS-1 materials. Fibroblasts from L929 cell line (ATCC, CCL-1, ATCC, Manassas, VA, USA) were cultured in contact with DS-1 electrospun polyimide fibers at a density of $2 \times 10^{4}$ cells $/ \mathrm{cm}^{2}$ and monitored for $24 \mathrm{~h}$ to allow cell adhesion and forming of cell-material constructs. For biocompatibility assessment, these constructs were permanently compared to L929 culture on TCPS, which was used as a control. Biocompatibility evaluation comprised of viability and proliferation analysis via MTT test, DS-1 cytotoxicity analysis by LDH assay, and visualization of live and dead cells in the culture by Live/Dead assay. 
MTT assay is a quantitative method which allows evaluation of cells ${ }^{\prime}$ metabolic activity. Briefly, L929 murine fibroblasts were incubated with $1 \mathrm{mg} / \mathrm{mL}$ [3-(4.5-dimethylthiazol-2yl)]-2.5diphenyltetrazolium bromide (MTT) solution for $4 \mathrm{~h}$ in the dark, at $37{ }^{\circ} \mathrm{C}$. After incubation, formazan crystals were solubilized with isopropanol, resulting in purple solution, quantified by spectrophotometry at $550 \mathrm{~nm}$, using FlexStation3 (Molecular Devices, Foster City, CA, USA).

Electrospun polyimide fibers' cytotoxicity exerted on the cells was investigated using LDH test (Tox7 kit, Sigma-Aldrich), according to manufacturer's instructions-cells that no longer have membrane integrity release lactate dehydrogenase (LDH) into the culture medium. In our experiment, the medium was collected and mixed with the kit's components in order to be evaluated after two and six days of culture by spectrophotometric measurement at $490 \mathrm{~nm}$.

Live/Dead assay is a qualitative test, based on two components revealing simultaneously stain live and dead cells and allowing visualization of cell dispersion and morphology inside the materials. The test was assessed following manufacturer's protocol using Live/Dead kit (ThermoFisher Scientific, Foster City, CA, USA). The kit includes calcein AM-staining cells that have an intact cell membrane (live cells) in green and ethidium bromide-staining cells with a damaged membrane integrity (dead cells) in red. Images were obtained using confocal microscopy (Carl Zeiss LSM 710, Jena, Germany) and then processed with Zeiss Zen 2010 software.

Statistical analysis was performed using Graph Pad Prism 6.0 software, One-Way ANOVA and Bonferroni correction. Statistically significant values were considered for $p<0.05$.

\section{Results and Discussion}

The chosen DS-1 polyimide have been synthesized by the classic two steps polycondensation reaction, using equimolecular amounts of 4,4'-diamino-4"-hydroxytriphenylmethane and 4,4'-oxydiphthalic anhydride as it was depicted in Scheme 1, following a detailed method described elsewhere [33]. The synthesized DS-1 polyimide was soluble in common organic solvent such as dimethyl sulfoxide (DMSO), $N, N$-dimethylformamide (DMF), $N, N$-dimethylacetamide (DMAc), and $N$-methyl-2-pyrrolidone (NMP). The good solubility of DS-1 was attributed to the presence of the pendant phenol groups in the structural unit, which reduced the inter-chain interactions and thus increasing the solubility. The chemical structure of DS-1 polyimide was confirmed by the ATR-FTIR and ${ }^{1} \mathrm{H}$ NMR spectroscopy. The complete imidization process was confirmed by the presence, in the FTIR spectrum, of imide characteristic absorption bands located at $1773 \mathrm{~cm}^{-1}$, attributed to $\mathrm{C}=\mathrm{O}$ asymmetric stretching vibration, $1720 \mathrm{~cm}^{-1}$, attributed to $C=O$ symmetric stretching vibration, and $745 \mathrm{~cm}^{-1}$, attributed to $\mathrm{C}-\mathrm{N}$ ring deformation. Moreover, the chemical structure of DS-1 polyimide was confirmed by ${ }^{1} \mathrm{H} \mathrm{NMR}$, with complete assignment of the protons as it was listed in the experimental section.

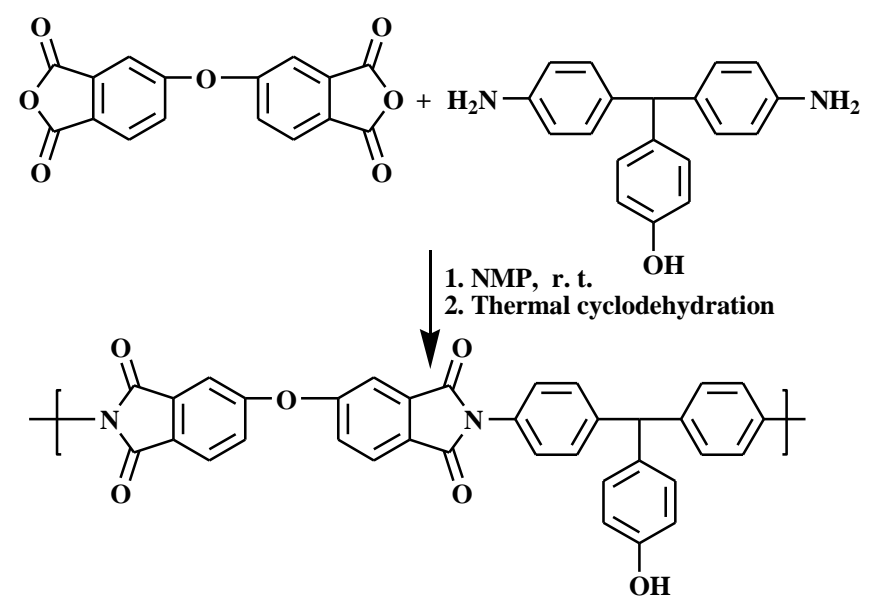

Scheme 1. Schematic pathway to describe the synthesis of polyimide DS-1. 
Further in this study, we performed cytotoxicity tests on a membrane based on electrospun polyimide fibers, obtained from DS-1 polyimide, developed for various biomedical applications, for example: wound dressing, pharmaceutical patches, bandages or meshes, etc. In this respect, facile production of hydroxy-functional electrospun polyimide fibers, through electrospinning process, is described further. All electrospinning parameters such as polymer concentration, solvents, flow rate, the distance between drum and jet were optimized according to our previously reported work [44]. Thus, following the rationale optimization procedure of electrospinning process, largely described in the above cited paper, the optimal condition to obtain DS-1 polyimide fibers having small diameters and narrow distribution without beads were found to be: $23 \mathrm{wt} \%,+10 \mathrm{kV} /-2 \mathrm{kV}$ dual voltage, $10 \mathrm{~cm}$ spinning distance at $5 \mu \mathrm{L} / \mathrm{min}$ feed rate. Using these parameters, the average fibers diameters for the electrospun polyimide fibers was found to be equal with $730 \pm 89 \mathrm{~nm}$. As shown in Figure 1, uniform, smooth, and bead free fibers were produced.

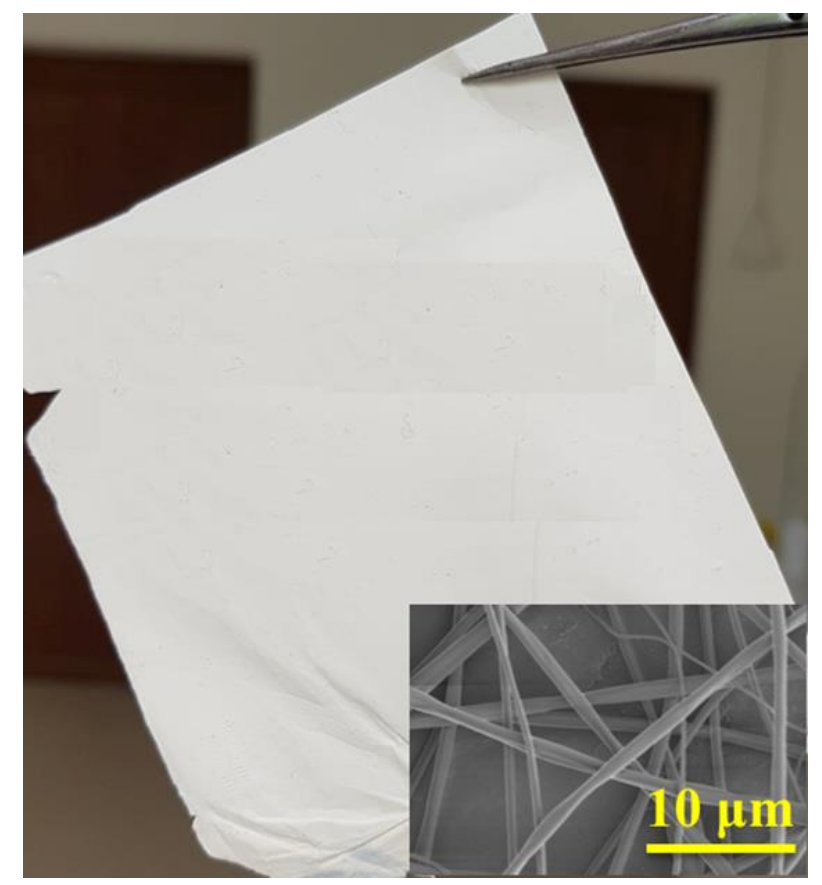

Figure 1. Image showing large scale membrane and SEM image of the electrospun polyimide fibers obtained by electrospinning process.

Reference Raman spectra obtained from electrospun polyimide fibers contained several distinctive bands. Figure 2 shows the averaged reference Raman of electrospun polyimide fibers while the left corner up inset display 50x optical image of electrospun polyimide fibers. Raman vibration spectroscopies were taken to assess the nature of chemical bonding, interactions, conformations, and even orientations of molecules in electrospun polyimide fibers. In the high-wavenumber spectral region (3050-3500 $\mathrm{cm}^{-1}$ ) of the Raman spectrum, two low-intensity bands attributed to the O-H stretching modes are present at 3366 and $3198 \mathrm{~cm}^{-1}$. Characteristic bands for polyimide are imide $\mathrm{I}(\mathrm{C}=\mathrm{O}$ stretch at $\left.1789 \mathrm{~cm}^{-1}\right)$, imide II (C-N-C axial vibration stretch at $\left.1385 \mathrm{~cm}^{-1}\right)$, imide III (C-N-C transverse vibration stretch at $\left.1125 \mathrm{~cm}^{-1}\right)$. These detected functional bands indicated that the electrospun polyimide fibers were highly imidized under the curing conditions used. The bands near $1630 \mathrm{~cm}^{-1}$ and $1161 \mathrm{~cm}^{-1}$ were attributed to ring stretching modes of benzene rings in the polyimide backbone. The bands at 363 , 486 , and $552 \mathrm{~cm}^{-1}$ can be attributed to $\mathrm{C}-\mathrm{O}-\mathrm{C}$ stretching. The morphology of electrospun polyimide fibers was confirmed by Raman spectroscopy (Figure 2, left corner up inset). 


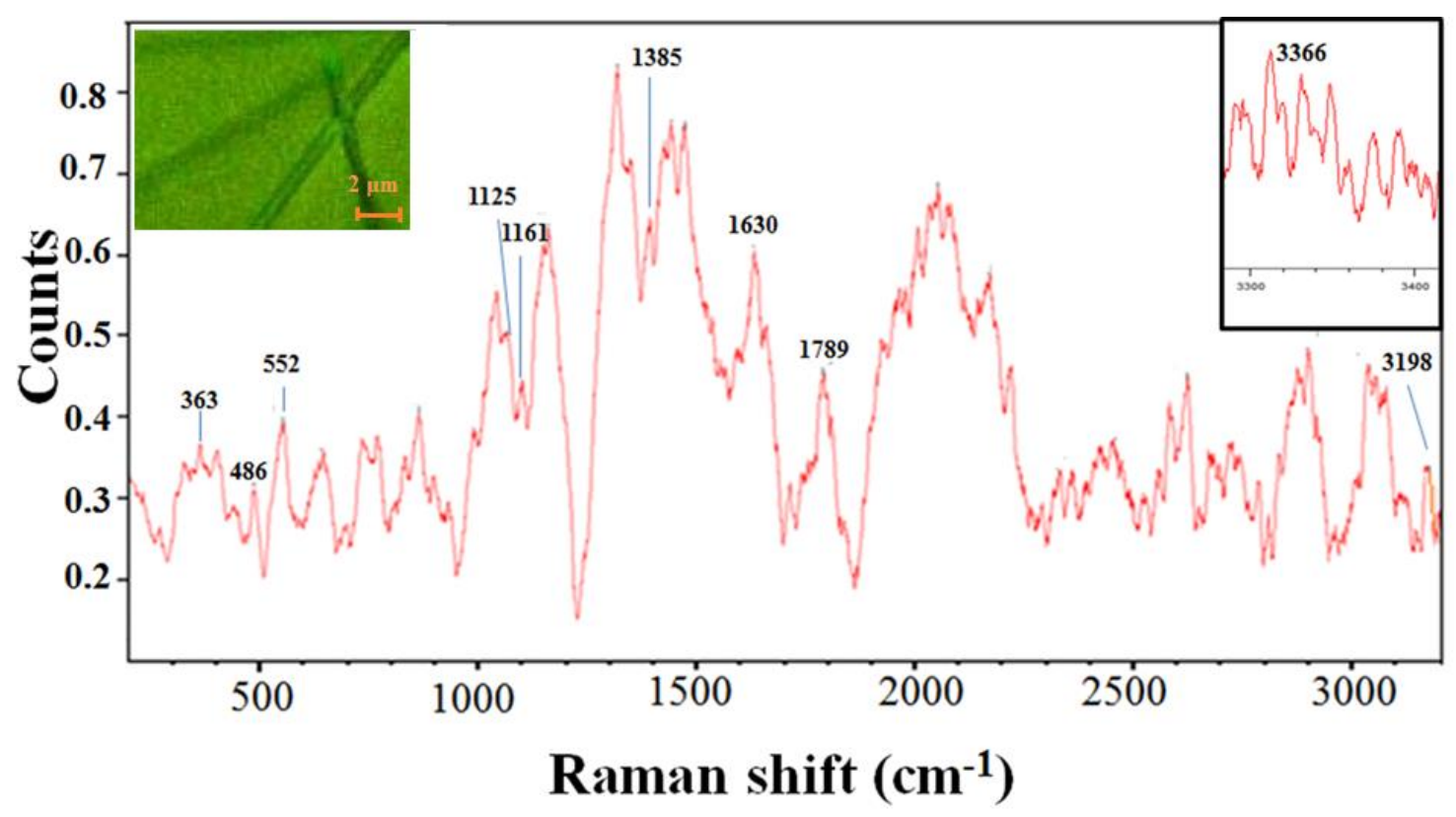

Figure 2. Raman spectrum and Raman image (left corner up inset) obtained by Raman spectroscopy measurements on the DS- 1 electrospun polyimide fibers.

It has been noticed in the literature that polyimides are generally biocompatible $[26,46-48]$. However, freestanding and flexible polyimide films or membranes suffer from the point of view of interaction properties of the polymer substrate with the cells. These interactions are essential for targeted biomedical applications such as transdermal therapy, bioactive wound dressing, or dermal repair/regeneration. Some surface modification processes were designed to incorporate certain functional groups, e.g., amino, hydroxyl or methyl, in order to influence the non-specific interactions of the protein with the surface and thereby influence cellular interaction. Thus, our interest here was not only to provide a polyimide substrate in the form of an electrospun fibers membrane, but also, to design a chemical structure for the chosen polyimide in such manner that it contains free functional hydroxyl groups, without altering the substrate by surface modification procedures.

It is generally known that surface wettability is an important parameter of membranes for both fundamental and practical applications [38]. Surface wettability influences biological response of the biomaterials for application in biomedicine. For example, wettability can be responsible for some capabilities of wound dressing materials, such as the ability to absorb exudates, the regulations of moist environment, and stimulation of the cell regeneration rate [49]. The role of wettability in protein adsorption, blood coagulation, cell and bacterial adhesion, etc. has been demonstrated in several studies [50-52]. Moreover, the dynamics of the surface of a material can be described by means of contact angle measurements because the surface layer is examined at a depth comparable to the radius of action of the interfacial forces involved in the surface restructuring [53].

To investigate the effect of the presence of free hydroxyl functional groups on wettability properties of DS-1 polyimide, water contact angle and water uptake capacity of DS-1 fibers membrane were measured with obtained results being presented in Figure 3. The water contact angle of the electrospun polyimide fibers was $>90^{\circ}$ (Figure $3 a$, left corner up inset), the value of $108.79 \pm 0.5^{\circ}$ was indicative of a typical hydrophobic feature. In general, it has been shown in the literature that surface wettability of electrospun fiber materials is associated with a variety of factors, which include the nature of the polymeric material, and some characteristics related to the structure of the surface at nano-micro level, such as roughness, fiber diameter, pore size, and porosity [54]. One reason for hydrophobic behavior of DS-1 electrospun fibers could be explained by its varying microstructure and large specific surface. However, there was a reasonable drop in the water contact angle for DS-1 fibers membrane, 
in comparison with other polyimide structures reported in the literature [53] reflecting the advantage of the presence of OH groups along the DS-1 macromolecule [54].

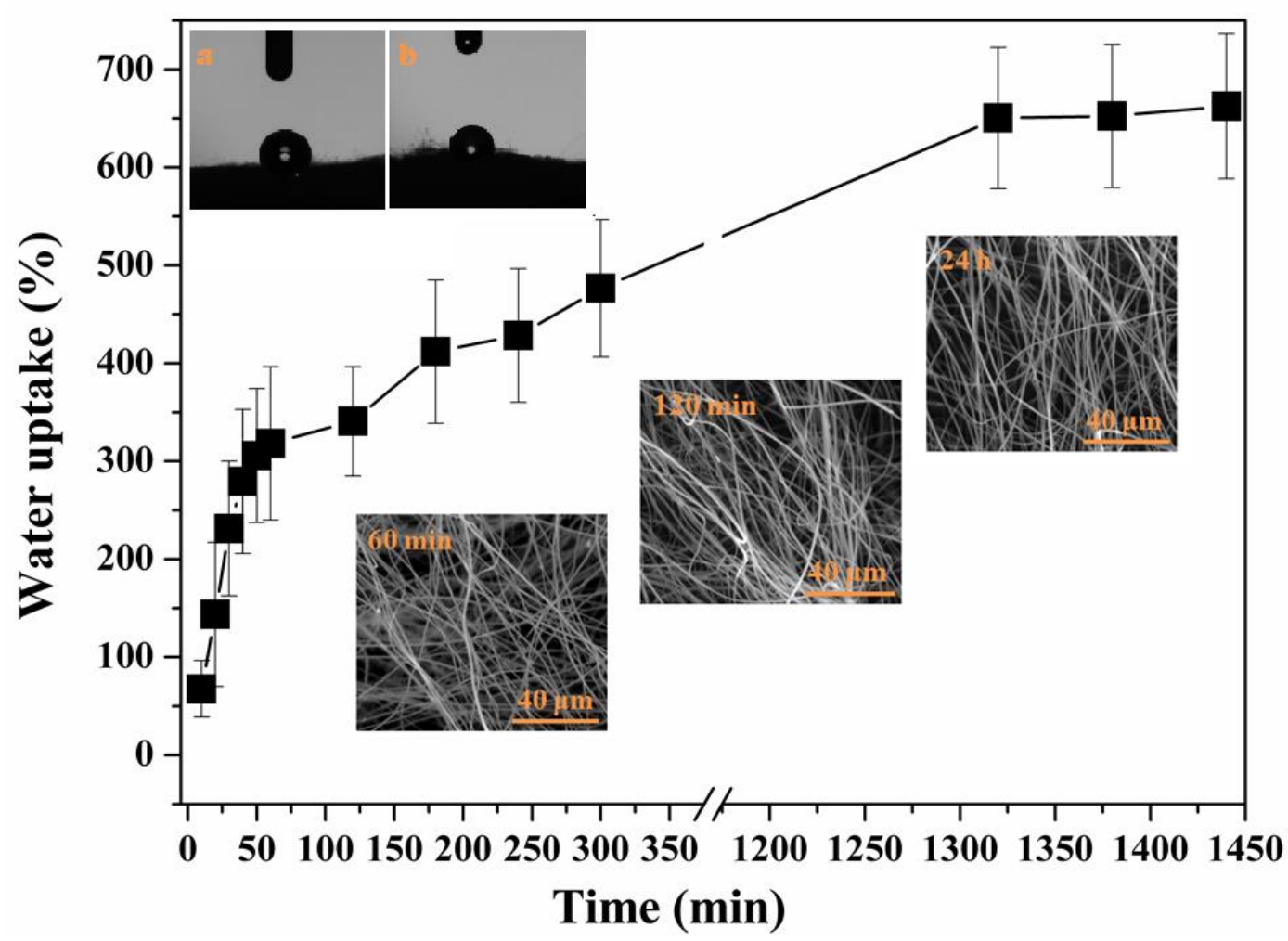

Figure 3. Water uptake capacity of DS-1 fibers, and SEM images of the samples after swelling periods of $60 \mathrm{~min}, 120 \mathrm{~min}$ and $24 \mathrm{~h}$, respectively. In the left corner up insets, images from the contact angle experiments on the sample before swelling (a) and after swelling study (b), are given.

To act as a promising wound dressing material, an electrospun fibers membrane should display exudate soaking ability, wound wetting capacity, non-adherence, easy to remove ability [55]. The properties can be determined through water uptake capacity. In this study, the absorption capability of DS-1 polyimide fibers membrane was studied at different time points from $10 \mathrm{~min}$ to $24 \mathrm{~h}$. The DS- 1 membrane was found to have an impressive absorption percentage of $620 \%$ after $24 \mathrm{~h}$ of incubation. This significant enhancement can be attributed to the availability of $\mathrm{OH}$ functional groups. Moreover, the porous nature of DS-1 fibrous membrane may also have contributed to the equilibrium absorption percentage by supporting the diffusion of more water molecules into the fiber network. In order to test the dimensional stability of DS-1 fibers after swelling experiments, the dry samples corresponding to $60 \mathrm{~min}, 120 \mathrm{~min}$, and $24 \mathrm{~h}$ of swelling were subjected to SEM investigation. As it can be observed in Figure 3, all the images, corresponding to three different time periods of swelling, exhibited similar fibrous morphology for the studied samples, as the non-swollen DS-1 fibers. To complement these observations, contact angle measurements were performed on the sample subjected to the swelling experiments. After $24 \mathrm{~h}$ of swelling, the sample was allowed to dry, then, its contact angle value was measured. Unfortunately, the planarity of the membrane is a little bit affected after the swelling process, thus, the new contact angle increased slightly $\left(112.79 \pm 0.23^{\circ}\right.$, Figure $3 b$, left corner up inset), probably due to the modification of surface topography.

The swelling absorption results indicated that the currently studied electrospun fiber membrane, based on polyimide having free-OH functional groups, provided broader hydrophilic sites inside the polymer matrix and higher surface area for water absorption. Thus, from practical point of view, this would not only prevent the loss of the fluids and nutrients from the human body in the in vivo tests, but would also increase the cell attachment and proliferation rate of fibrous polyimide membrane [56], 
which is advantageous for targeted specific applications such as bioactive wound dressing material, medical/pharmaceutical patches or bandages and other.

The physicochemical stability of the studied DS-1 polymeric matrix is an important parameter when looking for potential biomedical applications of this material. In order to check if the morphology and the integrity of the structure of electrospun polyimide fibers could be affected by the PBS solution, small pieces of DS-1 samples $(1 \mathrm{~cm} \times 1 \mathrm{~cm})$ were immersed in the PBS solution for a period of two and six days, respectively. After washing and drying these samples they were subjected to SEM investigation (Figure 4). As it has can be seen in the images taken at $100 \mu \mathrm{m}$ magnification the electrospun polyimide fibers keep the morphology of their fibrous structure after immersing the samples in PBS either for two or six days, respectively. The average diameter of the electrospun polyimide fibers was calculated and it was observed that the average diameter was not affected by the presence of PBS solution (730 $\pm 89 \mathrm{~nm}$ ) (the insets micrographs at $40 \mu \mathrm{m}$ magnification). In order to complement these findings, after performing the swelling experiments, the samples were allowed to dry in an oven for $36 \mathrm{~h}$ at $60^{\circ} \mathrm{C}$, then, they were weighed to confirm their initial masses, as a parameter for the morphological stability, and structural integrity of the DS-1 fibers membrane.
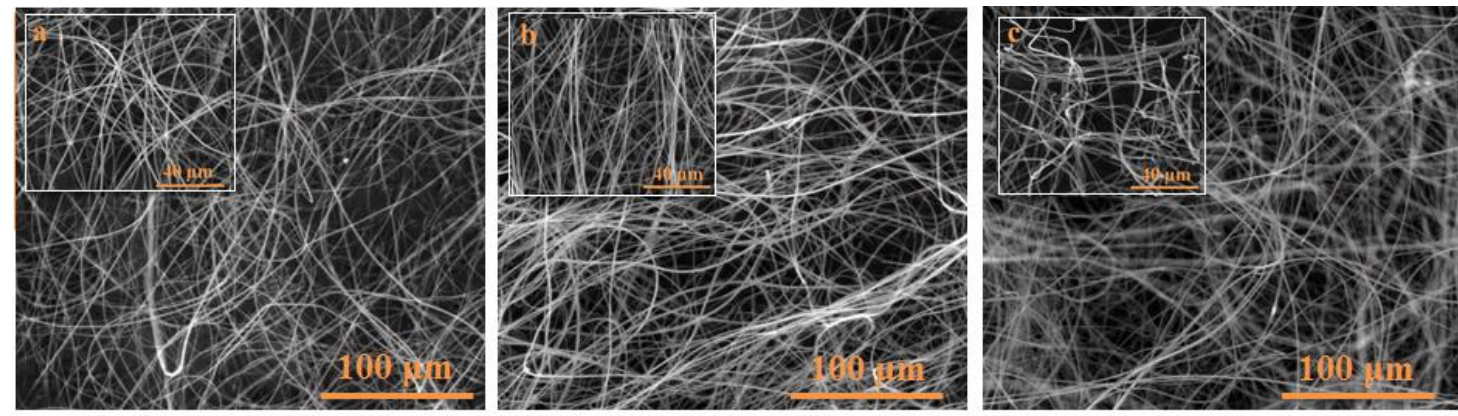

Figure 4. SEM micrographs of the electrospun polyimide fibers (a), electrospun polyimide fibers exposed to PBS (Phosphate-Buffered Saline) for two days (b) and six days (c) at $100 \mu \mathrm{m}$ magnification and $40 \mu \mathrm{m}$, respectively.

In the next step of our study, the antibiofilm potential of electrospun polyimide fibers was examined against biofilm producing bacteria. We investigated the adherence of S. aureus and P. aeruginosa ATCC strains to electrospun polyimide fibers. Figure 5 demonstrates the very good antibiofilm properties of electrospun polyimide fibers compared to the controls (represented by tissue culture plastic control as well as urinary catheter). The antimicrobial behavior of electrospun polyimide fibers is more pronounced in the case of $S$. aureus compared to P. aeruginosa and it can be explained considering the presence of the pendant phenolic $\mathrm{OH}$ groups in the polyimide structure.

We chose a urinary catheter as positive control because when used in a clinical setting, it connects the heavily colonized perineum with the normally sterile bladder and therefore provides an entry route for microorganism multiplication and biofilm formation. S. aureus as well as P. aeruginosa produce on urinary catheters biofilms hard to eradicate in clinical practice. In our experiment, urinary catheters were colonized in $24 \mathrm{~h}$ by $3 \times 10^{5} \mathrm{CFUs}$ of P. aeruginosa and $10^{5} \mathrm{CFUs}$ of $S$. aureus. Compared to the urinary catheter control, the novel polyimide electrospun fibers membrane developed within this study showed a two-log reduction in bacterial growth for both $S$. aureus and $P$. aeruginosa $\left({ }^{*} P<0.05,{ }^{* *} P<0.01\right.$, ${ }^{*} P<0.001, \mathrm{n}=3, \pm$ S.E.M). In addition, DS-1 material proved to have an enhanced antibiofilm activity compared to the TCPS control ( $p=0.0375$ for $S$. aureus, $p=0.0016$ for $P$. aeruginosa).

MTT assay results, after two days of culture in standard conditions, showed an overall good viability of L929 cultured in contact with both control system (CTRL) and the DS-1 material. The DS-1 material indicated a slightly increased viability (Figure 6) compared to the TCPS control value, although no statistical significance was observed. After the cells were maintained in culture for six days with the electrospun polyimide fibers, the higher viability tendency observed at two days became obvious. Hence, the highest statistically significant $(p<0.05)$ increased viability rate was noticed for the cells 
cultured in contact with the DS-1 composite in comparison to the control system. The results also indicated a statistically significant $(p<0.01)$ increased cell proliferation after six days of culture on DS-1. Since viability and proliferation were better on the DS-1 material than on TCPS control, this might suggest that the electrospun polyimide fibers membrane did not affect the fibroblasts cell viability in the evaluated time points, probably due to material's high surface area to volume ratio.

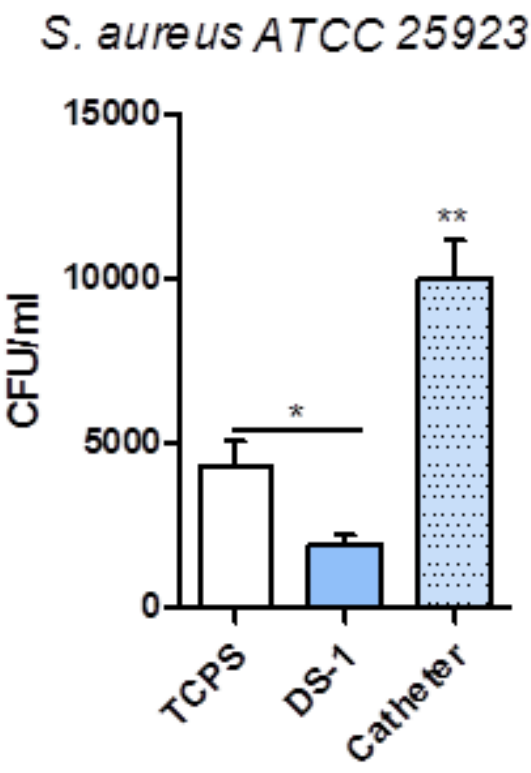

(a)
$P$. aeruginosa ATCC 27853

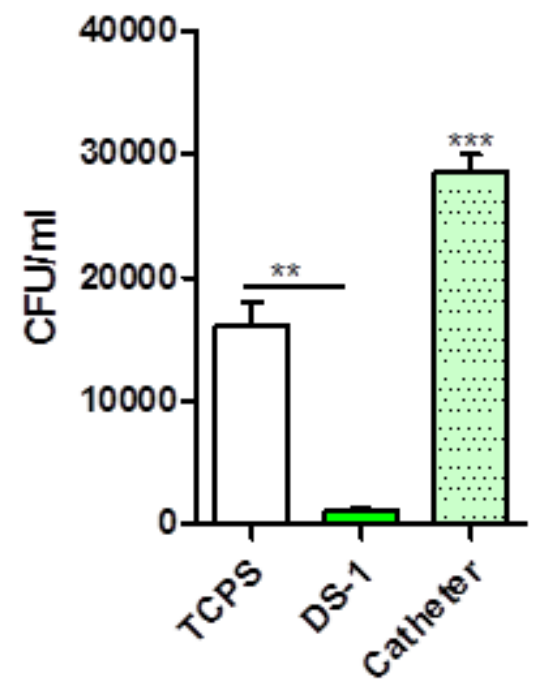

(b)

Figure 5. Strains adherence of S. aureus and P. aeruginosa (ATCC strains) to electrospun polyimide fibers. (a) note; (b) note.

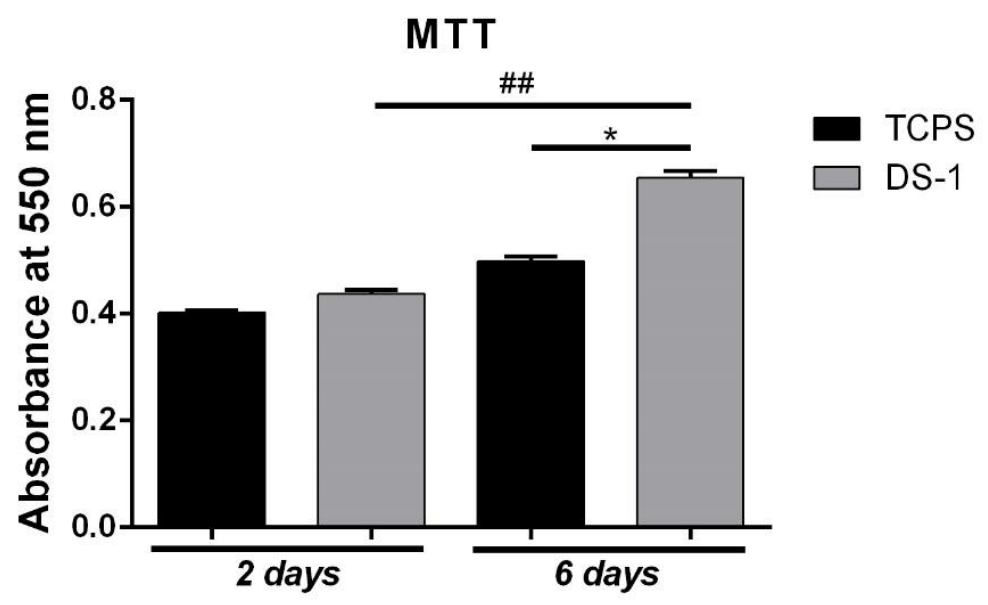

Figure 6. Cell viability and proliferation profile registered for DS-1 after two and six days of culture, evaluated by MTT assay. Statistical significance: ${ }^{*} p<0.05$ (DS-1 six days versus TCPS six days); \#\# $p<0.01$ (DS-1 six days versus DS-1 two days).

After two days of culture in standard conditions, LDH assay indicated a similar cytotoxic effect (Figure 7) for DS-1 material as for TCPS, since a low number of dead cells were found after they were kept in contact with the materials. At six days, the cytotoxicity of the DS-1 composite slightly increased, but no statistical significance was observed between its cytotoxicity and the one of TCPS control. The results suggest that electrospun polyimide fibers did not exert a significant cytotoxic effect on the cellular component. 


\section{LDH}

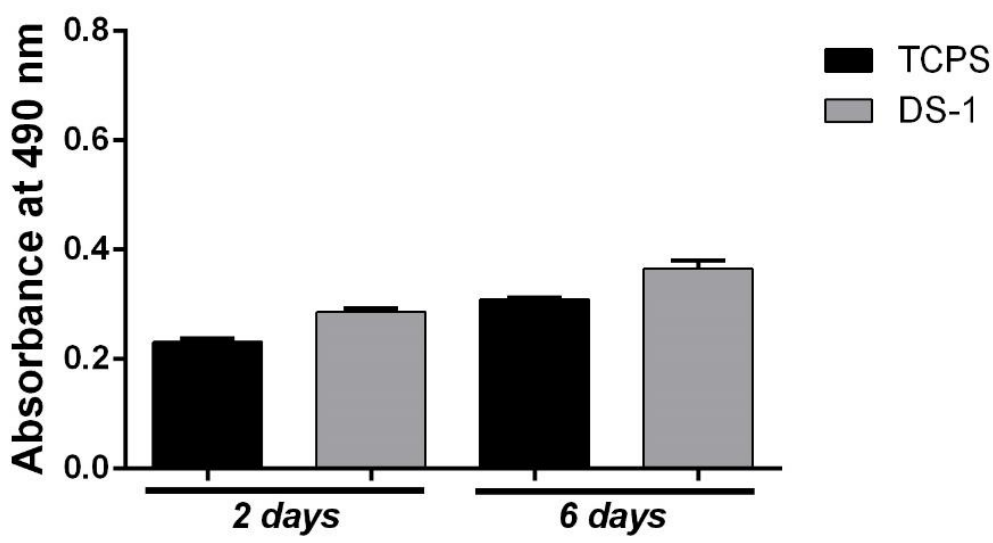

Figure 7. Cytotoxic level registered for DS-1 after two and six days of culture, evaluated by LDH assay.

Live/Dead staining supports the results of the MTT and LDH assays, given that the number of viable cells was considerably higher than the number of dead cells. The number of live cells was elevated on the DS-1 material compared to the control, after two days of culture in standard conditions. Six days after cell seeding, a great number of viable cells was observed on the DS-1 material in comparison to TCPS. Furthermore, confocal microscopy allowed the visualization of their tendency to proliferate on the electrospun polyimide fibers (Figure 8). These results could be due to the extremely interconnected pore network and thin fiber diameters in the fibers material that mimic the extracellular matrix environment.

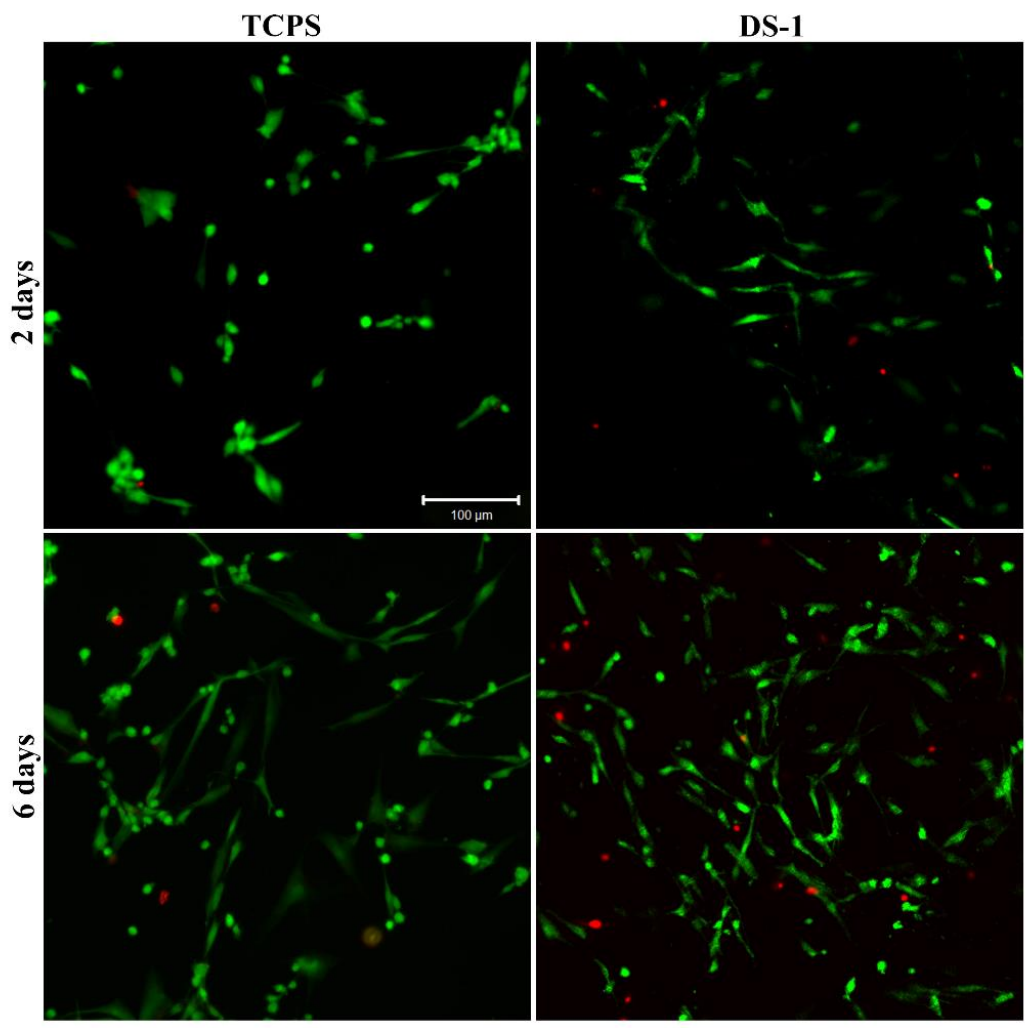

Figure 8. Confocal microscopy of live (green) and dead (red) cells cultured in contact with DS-1 for two and six days. 


\section{Conclusions}

The current study presents the preparation of electrospun fibers membrane based on a polyimide carrying hydroxyl functional groups. Structure-properties correlations related to water uptake capacity and water contact angle have been made. The limitation of the relatively high value of the contact angle could be overcome by further design of the chemical structure of the chosen polyimide. The cytotoxicity of the prepared membrane, evaluated by the MTT assay and Live/Dead ${ }^{\circledR}$ (Molecular Probes) assay, revealed high rates of viability and proliferation of fibroblast cells in contact with the electrospun polyimide fibers. Antibacterial and anti-biofilm activities of electrospun polyimide fibers against Staphylococcus aureus and Pseudomonas aeruginosa strains were investigated, resulting in aggregation of bacteria, and a reduction in biofilm development. According to LDH assay, after two days of culture in standard conditions, a high proportion of live cells has been observed on the tested samples and this proportion was even higher after six days of culture. All the obtained results imply further studies on this type of materials in order to establish their mechanical properties and to target specific biomedical application, such as wound dressing material, medical/pharmaceutical patches or bandages etc., at the interface between the material and the cells or living tissues.

Author Contributions: Conceptualization, D.S. and M.A.; Investigation, I.S., S.D., and D.R.; Methodology, T.V.-B. and G.G.P.; Software, T.V.-B., D.R., and G.G.P.; Supervision, D.S. and M.A.; Writing—original draft, D.S. and M.A.; Writing-review and editing, T.V.-B., D.R., G.G.P., I.S., and S.D.

Funding: This research was funded by European Regional Development Fund, grant number 86/8.09.2016, SMIS 105689.

Acknowledgments: The authors acknowledge the financial support of this research through the Project "Partnerships for knowledge transfer in the field of polymer materials used in biomedical engineering" IDP_40_443, Contract no. 86/8.09.2016, SMIS 105689, co-financed by the European Regional Development Fund by the Competitiveness Operational Programme 2014-2020, Axis 1Research, Technological Development and Innovation in support of economic competitiveness and business development, Action 1.2.3 Knowledge Transfer Partnerships.

Conflicts of Interest: The authors declare no conflict of interest.

\section{References}

1. Choong, P.F.M.; Dowsey, M.M.; Carr, D.; Daffy, J.; Stanley, P. Risk factors associated with acute hip prosthetic joint infections and outcome of treatment with a rifampinbased regimen. Acta Orthop. 2007, 78, 755-765. [CrossRef] [PubMed]

2. Peersman, G.; Laskin, R.; Davis, J.; Peterson, M. Infection in Total Knee Replacement: A Retrospective Review of 6489 Total Knee Replacements. Clin. Orthop. Relat. Res. (1976-2007) 2001, 392, 15-23. [CrossRef]

3. Frieri, M.; Kumar, K.; Boutin, A. Antibiotic resistance. J. Infect. Public Health 2017, 10, 369-378. [CrossRef] [PubMed]

4. Agnes Mary, S.; Giri Dev, V.R. Electrospun Herbal Nanofibrous Wound Dressings for Skin Tissue Engineering. J. Text. Inst. 2014, 106, 886-895. [CrossRef]

5. Joshua, B.S.; Matthews, K.H.; Howard, N.E.S.; Gillian, E.M. Wound healing dressings and drug delivery systems: A review. J. Pharm. Sci. 2008, 97, 2892-2923.

6. Jouybar, A.; Seyedjafari, E.; Ardeshirylajimi, A.; Zandi-Karimi, A.; Feizi, N.; Khani, M.M.; Pousti, I. Enhanced Skin Regeneration by Herbal Extract-Coated Poly-L-Lactic Acid Nanofibrous Scaffold. Artif. Organs 2017, 41, E296-E307. [CrossRef]

7. Zhang, W.; Ronca, S.; Mele, E. Electrospun Nanofibres Containing Antimicrobial Plant Extracts. Nanomaterials 2017, 7, 42. [CrossRef] [PubMed]

8. Suganya, S.; Senthil Ram, T.; Lakshmi, B.S.; Giridev, V.R. Herbal drug incorporated antibacterial nanofibrous mat fabricated by electrospinning: An excellent matrix for wound dressings. J. Appl. Polym. Sci. 2011, 121, 2893-2899. [CrossRef]

9. Agarwal, S.; Wendorff, J.H.; Greiner, A. Use of electrospinning technique for biomedical applications. Polymer 2008, 49, 5603-5621. [CrossRef]

10. Al-Enizi, A.M.; Zagho, M.; Elzatahry, A.A. Polymer-Based Electrospun Nanofibers for Biomedical Applications. Nanomaterials 2018, 8, 259. [CrossRef] 
11. Bhattarai, R.S.; Bachu, R.D.; Boddu, S.H.S.; Bhaduri, S. Biomedical Applications of Electrospun Nanofibers: Drug and Nanoparticle Delivery. Pharmaceutics 2018, 11, 5. [CrossRef] [PubMed]

12. Manea, L.R.; Bertea, A.; Popa, A.; Bertea, A.P. Electrospun Membranes for Environmental Protection. Iop Conf. Ser. Mater. Sci. Eng. 2018, 374, 012081. [CrossRef]

13. Suja, P.S.; Reshmi, C.R.; Sagitha, P.; Sujith, A. Electrospun Nanofibrous Membranes for Water Purification. Polym. Rev. 2017, 57, 467-504. [CrossRef]

14. Kadam, V.V.; Wang, L.; Padhye, R. Electrospun nanofibre materials to filter air pollutants-A review. J. Ind. Text. 2016, 47, 2253-2280. [CrossRef]

15. Yang, E.; Xu, Z.; Chur, L.K.; Behroozfar, A.; Baniasadi, M.; Moreno, S.; Huang, J.; Gilligan, J.; Minary-Jolandan, M. Nanofibrous Smart Fabrics from Twisted Yarns of Electrospun Piezopolymer. ACS Appl. Mater. Interfaces 2017, 9, 24220-24229. [CrossRef]

16. Sazanov, Y.N. Applied Significance of Polyimides. Russ. J. Appl. Chem. 2001, 74, 1253-1269. [CrossRef]

17. Sroog, C.E. Polyimides. Prog. Polym. Sci. 1991, 16, 561-694. [CrossRef]

18. Zhang, F.; Saleh, E.; Vaithilingam, J.; Li, Y.; Tuck, C.J.; Hague, R.J.M.; Wildman, R.D.; He, Y. Reactive material jetting of polyimide insulators for complex circuit board design. Addit. Manuf. 2019, 25, 477-484. [CrossRef]

19. Park, S.J.; Lee, E.J.; Kwon, S.H. Influence of Surface Treatment of Polyimide Film on Adhesion Enhancement between Polyimide and Metal Films. Bull. Korean Chem. Soc. 2007, 28, 188-192.

20. Verdianz, T.; Simbürger, H.; Liska, R. Surface modification of imide containing polymers I: Catalytic groups. Eur. Polym. J. 2006, 42, 638-654. [CrossRef]

21. Verdianz, T.; Simbürger, H.; Liska, R. Surface modification of imide containing polymers II: Co-reactive groups. Eur. Polym. J. 2006, 42, 869-882. [CrossRef]

22. Choi, M.C.; Wakita, J.; Ha, C.S.; Ando, S. Highly Transparent and Refractive Polyimides with Controlled Molecular Structure by Chlorine Side Groups. Macromolecules 2009, 42, 5112-5120. [CrossRef]

23. Jackson, N.; Muthuswamy, J. Flexible Chip Scale Package and Interconnect for Implantable MEMS Movable Microelectrodes for the Brain. J. Microelectromech. Syst. 2009, 18, 396-404. [CrossRef] [PubMed]

24. Mercanzini, A.; Colin, P.; Bensadoun, J.; Bertsch, A.; Renaud, P. In Vivo Electrical Impedance Spectroscopy of Tissue Reaction to Microelectrode Arrays. IEEE Trans. Biomed. Eng. 2009, 56, 1909-1918. [CrossRef] [PubMed]

25. Kanno, M.; Kawakami, H.; Nagaoka, S.; Kubota, S. Biocompatibility of fluorinated polyimide. J. Biomed. Mater. Res. 2002, 60, 53-60. [CrossRef]

26. Richardson, R.R., Jr.; Miller, J.A.; Reichert, W.M. Polyimides as biomaterials: Preliminary biocompatibility testing. Biomaterials 1993, 14, 627-635. [CrossRef]

27. Lee, K.K.; He, J.; Singh, A.; Massia, S.; Ehteshami, G.; Kim, B.; Raupp, G. Polyimide-based intracortical neural implant with improved structural stiffness. J. Micromech. Microeng. 2003, 14, 32-37. [CrossRef]

28. Lago, N.; Yoshida, K.; Koch, K.P.; Navarro, X. Assessment of Biocompatibility of Chronically Implanted Polyimide and Platinum Intrafascicular Electrodes. IEEE Trans. Biomed. Eng. 2007, 54, 281-290. [CrossRef]

29. Van Vlierberghe, S.; Sirova, M.; Rossmann, P.; Thielecke, H.; Boterberg, V.; Rihova, B.; Schacht, E.; Dubruel, P. Surface Modification of Polyimide Sheets for Regenerative Medicine Applications. Biomacromolecules 2010, 11, 2731-2739. [CrossRef]

30. Ding, Y.; Hou, H.; Zhao, Y.; Zhu, Z.; Fong, H. Electrospun polyimide nanofibers and their applications. Prog. Polym. Sci. 2016, 61, 67-103. [CrossRef]

31. Jiang, S.; Hou, H.; Agarwal, S.; Greiner, A. Polyimide Nanofibers by "Green” Electrospinning via Aqueous Solution for Filtration Applications. ACS Sustain. Chem. Eng. 2016, 4, 4797-4804. [CrossRef]

32. Serbezeanu, D.; Popa, A.M.; Sava, I.; Carja, I.D.; Amberg, M.; Rossi, R.M.; Fortunato, G. Design and synthesis of polyimide-Gold nanofibers with tunable optical properties. Eur. Polym. J. 2015, 64, 10-20. [CrossRef]

33. Aflori, M.; Serbezeanu, D.; Carja, I.D.; Fortunato, G. Gold Nanoparticles Incorporated into Electrospun Polyimide Fibers. Chem. Lett. 2015, 44, 1440-1442. [CrossRef]

34. Serbezeanu, D.; Popa, A.M.; Stelzig, T.; Sava, I.; Rossi, R.M.; Fortunato, G. Preparation and characterization of thermally stable polyimide membranes by electrospinning for protective clothing applications. Text. Res. J. 2015, 85, 1763-1775. [CrossRef] 
35. Butnaru, I.; Serbezeanu, D.; Bruma, M.; Sava, I.; Gaan, S.; Fortunato, G. Physical and thermal properties of poly(ethylene terephthalate) fabric coated with electrospun polyimide fibers. High Perform. Polym. 2015, 27, 616-624. [CrossRef]

36. Serbezeanu, D.; Butnaru, I.; Varganici, C.D.; Bruma, M.; Fortunato, G.; Gaan, S. Phosphorus-containing polyimide fibers and their thermal properties. RSC Adv. 2016, 6, 38371-38379. [CrossRef]

37. Annabi, N.; Nichol, J.W.; Zhong, X.; Ji, C.; Koshy, S.; Khademhosseini, A.; Dehghani, F. Controlling the porosity and microarchitecture of hydrogels for tissue engineering. Tissue Eng. Part B Rev. 2010, 16, 371-383. [CrossRef]

38. Mohammadzadehmoghadam, S.; Dong, Y. Fabrication and Characterization of Electrospun Silk Fibroin/Gelatin Scaffolds Crosslinked With Glutaraldehyde Vapor. Front. Mater. 2019, 6, 91. [CrossRef]

39. Bernards, D.A.; Desai, T.A. Nanoscale porosity in polymer films: Fabrication and therapeutic applications. Soft Matter 2010, 6, 1621-1631. [CrossRef]

40. Wang, Q.; Song, W.L.; Wang, L.; Song, Y.; Shi, Q.; Fan, L.Z. Electrospun polyimide-based fiber membranes as polymer electrolytes for lithium-ion batteries. Electrochim. Acta 2014, 132, 538-544. [CrossRef]

41. Yi, B.; Zhao, Y.; Tian, E.; Li, J.; Ren, Y. High-performance polyimide nanofiber membranes prepared by electrospinning. High Perform. Polym. 2019, 31, 438-448. [CrossRef]

42. Sode, K.; Sato, T.; Tanaka, M.; Suzuki, Y.; Kawakami, H. Carbon nanofibers prepared from electrospun polyimide, polysulfone and polyacrylonitrile nanofibers by ion-beam irradiation. Polym. J. 2013, 45, 1210. [CrossRef]

43. Chen, P.; Zhang, W.A.; Luo, W.; Fang, Y. Synthesis of superabsorbent polymers by irradiation and their applications in agriculture. J. Appl. Polym. Sci. 2004, 93, 1748-1755. [CrossRef]

44. Serbezeanu, D.; Carja, I.D.; Sava, I.; Rossi, R.M.; Fortunato, G. Multiphase Polymer Systems. Chapter 16. Development of Imide-Type Polymer Fibers Containing Metal Nanoparticles, 1st ed.; Barzic, A.E., Ioan, S., Eds.; CRC Press: Boca Raton, FL, USA, 2017; p. 18. [CrossRef]

45. Geilich, B.M.; Webster, T.J. Reduced adhesion of Staphylococcus aureus to ZnO/PVC nanocomposites. Int. J. Nanomed. 2013, 8, 1177-1184.

46. Teo, A.J.T.; Inkyu Park, A.M.; Kim, Y.-J.; Park, W.-T.; Yoon, Y.-J. Polymeric Biomaterials for Medical Implants and Devices. ACS Biomater. Sci. Eng. 2016, 2, 4454-4472.

47. Del Valle, J.; de la Oliva, N.; Müller, M.; Stieglitz, T.; Navarro, X. Biocompatibility evaluation of parylene C and polyimide as substrates for peripheral nerve interfaces. In Proceedings of the 20157 th International IEEE/EMBS Conference on Neural Engineering (NER), Montpellier, France, 22-24 April 2015; pp. $442-445$.

48. Stieglitz, T.; Beutel, H.; Schuettler, M.; Meyer, J.U. Micromachined, Polyimide-Based Devices for Flexible Neural Interfaces. Biomed. Microdevices 2000, 2, 283-294. [CrossRef]

49. Kim, S.E.; Heo, D.N.; Lee, J.B.; Kim, J.R.; Park, S.H.; Jeon, S.H.; Kwon, I.K. Electrospun gelatin/polyurethane blended nanofibers for wound healing. Biomed. Mater. 2009, 4, 044106. [CrossRef]

50. Dowling, D.P.; Miller, I.S.; Ardhaoui, M.; Gallagher, W.M. Effect of Surface Wettability and Topography on the Adhesion of Osteosarcoma Cells on Plasma-modified Polystyrene. J. Biomater. Appl. 2011, 26, 327-347. [CrossRef]

51. Shin, S.; Seo, J.; Han, H.; Kang, S.; Kim, H.; Lee, T. Bio-Inspired Extreme Wetting Surfaces for Biomedical Applications. Materials 2016, 9, 116. [CrossRef]

52. Menzies, K.L.; Jones, L. The impact of contact angle on the biocompatibility of biomaterials. Optom. Vis. Sci. Off. Publ. Am. Acad. Optom. 2010, 87, 387-399. [CrossRef]

53. Wang, Y.X.; Robertson, J.L.; Spillman, W.B.; Claus, R.O. Effects of the chemical structure and the surface properties of polymeric biomaterials on their biocompatibility. Pharm Res. 2004, 21, 1362-1373. [CrossRef] [PubMed]

54. Balaji, A.; Jaganathan, S.K.; Ismail, A.F.; Rajasekar, R. Fabrication and hemocompatibility assessment of novel polyurethane-based bio-nanofibrous dressing loaded with honey and Carica papaya extract for the management of burn injuries. Int. J. Nanomed. 2016, 11, 4339-4355. 
55. Widgerow, A.D.; King, K.; Tocco-Tussardi, I.; Banyard, D.A.; Chiang, R.; Awad, A.; Afzel, H.; Bhatnager, S.; Melkumyan, S.; Wirth, G.; et al. The burn wound exudate-an under-utilized resource. Burns 2015, 41, 11-17. [CrossRef] [PubMed]

56. Razavi, S.; Karbasi, S.; Morshed, M.; Zarkesh Esfahani, H.; Golozar, M.; Vaezifar, S. Cell Attachment and Proliferation of Human Adipose-Derived Stem Cells on PLGA/Chitosan Electrospun Nano-Biocomposite. Cell J. 2015, 17, 429-437. [PubMed]

(C) 2019 by the authors. Licensee MDPI, Basel, Switzerland. This article is an open access article distributed under the terms and conditions of the Creative Commons Attribution (CC BY) license (http://creativecommons.org/licenses/by/4.0/). 